OPEN ACCESS

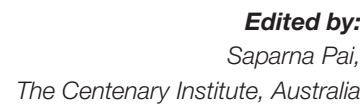

Reviewed by:

Samar S. Ayache,

Université Paris-Est, France

Julianne Teresa Djordjevic,

University of Sydney, Australia Luis R. Martinez,

New York Institute of Technology, USA

*Correspondence: Meiqing Shi mshi@umd.edu;

Christopher H. Mody cmody@ucalgary.ca

Specialty section: This article was submitted to Multiple Sclerosis and

Neuroimmunology,

a section of the journal

Frontiers in Immunology

Received: 15 April 2016

Accepted: 19 July 2016

Published: 02 August 2016

Citation:

Shi M and Mody CH (2016)

Fungal Infection in the Brain:

What We Learned from

Intravital Imaging.

Front. Immunol. 7:292.

doi: 10.3389/fimmu.2016.00292

\section{Fungal Infection in the Brain: What We Learned from Intravital Imaging}

\author{
Meiqing Shi ${ }^{* *}$ and Christopher H. Mody ${ }^{2,3 *}$ \\ ${ }^{1}$ Division of Immunology, Virginia-Maryland Regional College of Veterinary Medicine, University of Maryland, College Park, \\ MD, USA, ${ }^{2}$ Department of Microbiology, Immunology and Infectious Diseases, University of Calgary, Calgary, AB, Canada, \\ ${ }^{3}$ Department of Internal Medicine, University of Calgary, Calgary, AB, Canada
}

Approximately 1.2 billion people suffer from fungal diseases worldwide. Arguably, the most serious manifestation occurs when pathogenic fungi infect the brain, often causing fatal meningoencephalitis. For most fungi, infection occurs via the vascular route. The organism must first be arrested in the brain microvasculature and transmigrate into the brain parenchyma across the blood-brain barrier. As a result, host immune cells are recruited into the brain to contain the fungi. However, it remains poorly understood how fungi traffic to, and migrate into the brain and how immune cells interact with invading fungi in the brain. A new era of intravital fluorescence microscopy has begun to provide insights. We are able to employ this powerful approach to study dynamic interactions of disseminating fungi with brain endothelial cells as well as resident and recruited immune cells during the brain infection. In this review, with a focus on Cryptococcus neoformans, we will provide an overview of the application of intravital imaging in fungal infections in the brain, discuss recent findings and speculate on possible future research directions.

Keywords: intravital imaging, fungi, brain migration, immune cells, meningoencephalitis, $C$. neoformans

\section{INTRODUCTION}

Infectious meningitis and encephalitis are a major threat to human health, causing high mortality and morbidity throughout the world (1). Following infections, microbes including viruses, bacteria, fungi, and parasites can disseminate from sites of initial infection to the bloodstream. The circulating pathogens become arrested in the brain vasculature, followed by transmigration into the brain parenchyma across the blood-brain barrier (BBB). The BBB is a structural and functional barrier, which maintains the neural microenvironment by regulating the passage of molecules and cells into the brain (2). To date, three mechanisms have been proposed for pathogens to cross the BBB: transcellular migration, paracellular migration, and the Trojan horse mechanism (1). Once pathogens have translocated to the brain parenchyma, they proliferate and cause brain inflammation, often with devastating consequences. There are three fundamental questions in the field (Figure 1): (1) How are pathogens arrested in the brain vasculature? (2) How do pathogens migrate into the brain across the BBB? and (3) How do immune cells respond to the brain infection and do they clear the pathogen or cause inflammation in a constrained intracranial compartment that is highly susceptible to cellular dysfunction and increased pressure?

Modern advances in technology have provided opportunities to better understand hostpathogen interactions. Among them, imaging of organs in living animals, using high-resolution intravital microscopy (IVM), represents a major advance in the field. Using this technique, interactions of pathogens with brain endothelial cells, and their transmigration across the BBB can 


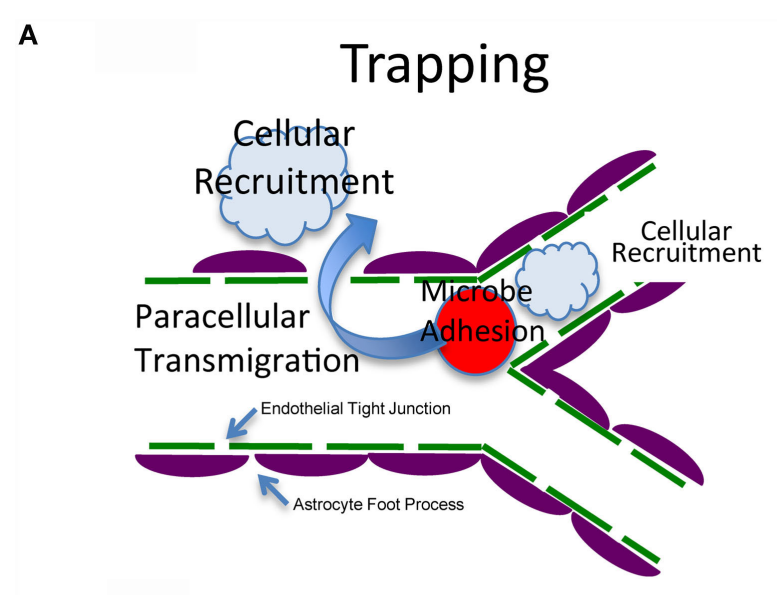

B

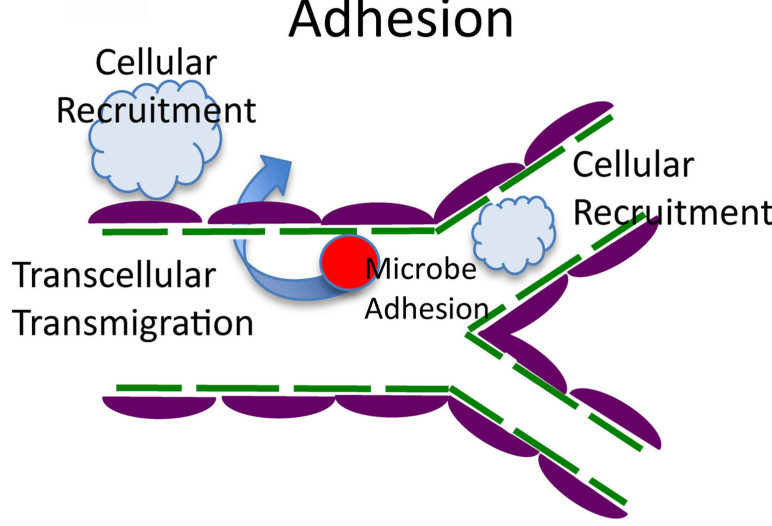

c Trojan Horse

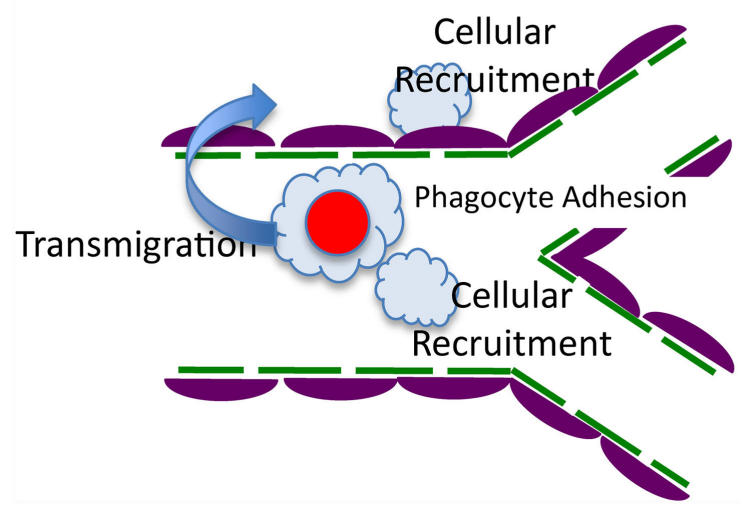

FIGURE 1 | Possible mechanisms of arrest, transmigration, and resultant host response. The BBB is formed by brain endothelial cells, which are connected by tight junctions, and astrocyte foot processes that surround the endothelial cells and maintain the integrity of the BBB $(2,3)$. (A) Fungal cells are trapped by vascular constriction with possible sensing and signaling of both cell types $(4,5)$. This is followed by transmigration that could be by a trans- or paracellular mechanism (paracellular is shown in this panel). Immune and inflammatory cells are recruited to the vascular or extracellular compartment to generate host defense and inflammation.

(B) Fungal cells adhere directly to the endothelium with possible sensing and

(Continued)

\section{FIGURE 1 | Continued}

signaling of both cell types $(6-10)$. This is followed by transmigration that could be by a trans- or paracellular mechanism (transcellular is shown in this panel). Immune and inflammatory cells are recruited to the vascular or extracellular compartment to generate host defense and inflammation. (C) Fungal cells are internalized within a host cell (Trojan Horse) that makes contact with the endothelium, arrests, and generates sensing and signaling of all three cell types $(11,12)$. This is followed by transmigration that could be by a trans- or paracellular mechanism. Immune and inflammatory cells are recruited to the vascular or extracellular compartment to generate host defense and inflammation.

be directly assessed under flow conditions in real time. In addition, the dynamic interactions of leukocytes with pathogens and their behavior in the brain vasculature and parenchyma can be evaluated in living animals. This is of particular importance, because the extravascular migration of pathogens and their interactions with immune cells are transient and highly dynamic, and investigation of these processes by direct observation using IVM provides insights that cannot be obtained using other techniques.

Of the approximately 300 fungal species that have been reported to be pathogenic to humans (13), Cryptococcus neoformans, Candida albicans, Histoplasma capsulatum, Coccidioides immitis, Paracoccidioides brasiliensis, Aspergillus spp., and zygomycetes are among the most common causes of brain or meningeal infections (14-21). In particular, cryptococcal meningoencephalitis is one of the most common infections of the central nervous system and a leading course of HIVassociated mortality globally $(16,18,22)$. In recent years, much progress has been made to understand migration of pathogens and immune responses induced by the invading pathogens in the brain using IVM. This review will discuss recent studies that used IVM to address brain infections by a very limited subset of pathogenic fungi (Table $\mathbf{1}$ ).

\section{INTRAVITAL MICROSCOPY}

Intravital microscopy was first employed by Julius Cohnheim in the nineteenth century to visualize leukocyte trafficking in the tongue and mesentery of a frog (27). In the last decade, significant progress has been made in imaging of live animals due to breakthroughs in microscopy. Wide-field microscopy, multiphoton confocal, spinning disk confocal, and multiphoton resonant scanning confocal microscopy have been used to image fungal infection in the brain. Each imaging system has its advantages or disadvantages depending on whether speed of image acquisition, depth into the tissue, image resolution, photobleaching and phototoxicity, and price are considerations (28-32).

\section{IVM PROCEDURE}

There are two major surgical methods to make the brain vasculature visible under fluorescent microscopy, i.e., a thinned-skull cranial window and an open-skull cranial window (33). Both techniques have advantages and limitations. During imaging through the thinned-skull cranial window, the brain does not 
TABLE 1 | Application of intravital imaging to brain infection by fungi.

\begin{tabular}{|c|c|c|c|}
\hline Fungi & Animals & Nature of the work & Reference \\
\hline C. neoformans & Mouse & $\begin{array}{l}\text { The fungal cell is mechanically } \\
\text { trapped in the brain capillary } \\
\text { and transmigrates to the brain } \\
\text { parenchyma with contributions } \\
\text { from urease }\end{array}$ & Shi et al. (5) \\
\hline C. neoformans & Mouse & $\begin{array}{l}\text { Neutrophils internalize the } \\
\text { intravascular fungal cell that } \\
\text { had been arrested in the brain } \\
\text { microvasculature and return to } \\
\text { the blood stream in a "vacuum- } \\
\text { cleaner" type of behavior }\end{array}$ & $\begin{array}{l}\text { Zhang } \\
\text { et al. (23) }\end{array}$ \\
\hline C. neoformans & Zebrafish & $\begin{array}{l}\text { The fungal cell was observed to } \\
\text { proliferate within macrophages; } \\
\text { capsule size determines early } \\
\text { macrophage control of infection }\end{array}$ & $\begin{array}{l}\text { Bojarczuk } \\
\text { et al. (24) }\end{array}$ \\
\hline C. neoformans & Zebrafish & $\begin{array}{l}\text { The fungal cell can cross the } \\
\text { zebrafish blood-brain barrier, } \\
\text { which is dependent on the FNX1 } \\
\text { virulence gene }\end{array}$ & $\begin{array}{l}\text { Tenor et al. } \\
(25)\end{array}$ \\
\hline C. albicans & Mouse & $\begin{array}{l}\text { Accumulation of both yeast and } \\
\text { filamentous forms of the fungal } \\
\text { cells were observed in the brain } \\
\text { meninges and parenchyma }\end{array}$ & $\begin{array}{l}\text { Navarathna } \\
\text { et al. (26) }\end{array}$ \\
\hline P. brasiliensis & Mouse & $\begin{array}{l}\text { Enhanced leukocyte recruitment } \\
\text { to the brain following the fungal } \\
\text { infection is associated with CXCL9 }\end{array}$ & $\begin{array}{l}\text { Pedroso } \\
\text { et al. (20) }\end{array}$ \\
\hline
\end{tabular}

need to be superfused with artificial cerebrospinal fluids because the brain tissue is still covered with the skull. It is well suited for observations over long periods of time. However, the skull thickness affects the image quality and achieving optimal and uniform skull thickness requires a high level of surgical proficiency. By contrast, in an open-skull window, a portion of the skull and dura is removed, and the cortical surface is directly exposed to microscopy. Thus, a better quality of images is usually achieved compared with a thinned-skull window. However, it is essential to superfuse the brain with artificial cerebrospinal fluid during the period of observation, and great care must be taken to avoid surgical trauma and hemorrhage (33).

To facilitate intravital imaging, the organisms, brain microvasculature, and leukocytes can be labeled with fluorochromes. For example, we labeled C. neoformans with fluorescent isothiocyanate (FITC) or tetramethylrhodamine isothiocyanate (TRITC) to visualize the arrest and migration of the yeast cell into the brain $(4,5)$. Two colors allow comparison of two different virulence characteristics or wild-type and mutant strains. However, the yeast cell loses the fluorescent label if it proliferates. This disadvantage might be overcome by using fungi expressing green or red fluorescent proteins if sufficient fluorescent intensity can be achieved $(26,34,35)$. To label the microvasculature, rat-anti-mouse PECAM-1 [CD31, a molecule expressed on endothelial cells (36)] can be injected intravenously $(37,38)$. Since the tight junctions of endothelial cells express high PECAM-1, this labeling can be used to study interactions of fungi or leukocytes with endothelial tight junctions (36). Alternatively, the vascular compartment can be illuminated by intravenous injection with fluorochrome-conjugated bovine serum albumin or dextran (39). In addition, transgenic mice that express fluorescent proteins in endothelial cells [for example, Tie-2 green fluorescent protein (GFP) mice (40)] can be used.

An expanding number of tools are becoming available to study the interactions of fungi with immune and inflammatory cells. To determine the trafficking of leukocytes in the brain, mice can be injected intravenously with rhodamine $6 \mathrm{G}$, which is a cellpermeant dye that is sequestered by active mitochondria $(41,42)$. However, to identify the functions of subsets of leukocytes, $\mathrm{mAb}$ or transgenic mice can be used. For example, anti-CD45 can be injected intravenously, which labels all leukocytes. Neutrophils can be labeled in vivo by intravenous injection of anti-Ly6G (23). Alternatively, neutrophils can be visualized in mice expressing enhanced GFP under the control of the endogenous lysozyme promoter (LysM-eGFP) $(39,43)$. To image monocytes, mice can be intravenously injected with fluorochrome-labeled anti-CCR2 (labels proinflammatory monocytes) or anti-CX3CR1 antibody (labels patrolling monocytes) (44). Alternately, CX3CR $1^{\text {gfp/ } /+}$ mice can be used to achieve this goal. In CX3CR1 $1^{\text {fp/ } /+}$ mice, one allele for the gene encoding CX3CR1, the receptor for chemokine CX3CL1, has been replaced with a gene encoding GFP, resulting in GFP expression of all circulating $\mathrm{CD} 11 \mathrm{~b}^{+} \mathrm{F} 4 / 80^{+}$cells. CX3CR $1^{\text {fpp/+ }}$ mice express GFP in monocytes, but not in neutrophils $(45,46)$. With time, many more mouse strains are becoming available that have fluorescent reporters linked to other genes that define different subsets of cells and allow us to study the role of those cells in the pathogenesis of infection.

\section{BRAIN INFECTION WITH C. NEOFORMANS}

Cryptococcus neoformans is an encapsulated budding yeast that causes a life-threatening illness in immunocompromised individuals, especially in AIDS patients. It is estimated that there are one million cases of cryptococcosis per year and 600,000 of these patients will die within 3 months of diagnosis (22). Cryptococcus is found in the environment and enters the body through the respiratory tract. Immunocompetent individuals are usually able to contain C. neoformans in the lung (47). In the case of an immunocompromised host, the yeast cells cannot be successfully contained and disseminate into the brain via the bloodstream, causing meningoencephalitis $(16,47)$.

Hematogenous dissemination of C. neoformans is one of the most critical steps in the development of meningoencephalitis. Prior to transmigration into the brain parenchyma, circulating C. neoformans must be arrested in the brain vasculature. We became interested in a number of questions related to the pathogenesis of cryptococcal meningoencephalitis. (1) Was C. neoformans arrested in the brain vasculature prior to transmigration and did the arrest occur in venules or capillaries? (2) How did C. neoformans behave during arrest? and (3) What was the mechanism(s) underlying the arrest of $C$. neoformans. As arrest of C. neoformans is a transient and dynamic process, we developed an in vivo model system based on IVM to study these questions $(4,5)$. We demonstrated that $C$. neoformans appeared in the mouse brain microvasculature within a few 
seconds after injection into the tail vein. When first seen, C. neoformans was moving with the same velocity as the blood, and no interaction of circulating $C$. neoformans with venular endothelial cells was observed. The number of yeast cells passing through postcapillary venules was greatest immediately after injection and gradually decreased over time. However, even after $18 \mathrm{~h}$, rare yeast cells could still be seen moving in the brain venules. C. neoformans appeared to move at the same speed as the blood and came to a sudden stop in the capillaries of the brain without rolling and tethering to the endothelial surface. Interestingly, the yeast cells were arrested in capillaries that appeared to be of the same or smaller diameter than the organism, often at branch points. Differences in viability, polysaccharide capsule (the major virulence factor), and strain failed to affect the deposition of the yeast cells. In particular, there was no significant difference in the behavior and the arrest of polystyrene microspheres of similar size in the brain capillary bed when compared with $C$. neoformans. These results suggest that $C$. neoformans is mechanically trapped in the brain, which raises novel challenges for therapies to avoid arrest.

Cryptococcus neoformans transmigrates into the brain parenchyma across the BBB after arrest in the brain capillaries. Previous studies, using in vitro techniques, have shown that $C$. neoformans can cross the endothelium of the brain via direct transcytosis $(6,48,49)$. It was further demonstrated that transcytosis is mediated by interactions between CD44 expressed on endothelium and cryptococcal hyaluronic acids $(7,8)$. A secreted fungal metalloprotease (9), an extracellular phospholipase B1 (10), and brain inositol (50) are critically involved in transcytosis of $C$. neoformans. In addition, it was also reported that $C$. neoformans invaded the brain via a "Trojan horse" mechanism with the help of phagocytes $(11,12)$. However, these studies have failed to determine the dynamics of BBB penetration by $C$. neoformans in the brain vasculature in vivo. Using IVM, we have recently characterized the transmigration of C. neoformans in vivo (5). Following arrest in the brain, C. neoformans was directly seen to cross the capillary wall of living animals in real time. In contrast to trapping, viability, but not replication, was required for $C$. neoformans to cross the $\mathrm{BBB}$. Urease is critically involved in brain transmigration of the organism. Accordingly, a urease inhibitor could ameliorate infection of the mouse brain by reducing transmigration of $C$. neoformans into the brain, suggesting that a therapeutic strategy aimed at inhibiting this enzyme might be beneficial in cryptococcal meningitis and encephalitis.

Arrest of $C$. neoformans in the brain vasculature led to questions about recognition of the organism by circulating leukocytes. Recently, we addressed this question with the use of IVM (23). Among all subsets of leukocytes in the circulation, neutrophils are the most abundant phagocytes and are usually the first immune cells to be recruited to a site of infection to eliminate pathogens (51). Early work had suggested that human neutrophils kill C. neoformans in vitro via an intracellular $(52,53)$ or extracellular killing mechanism (54). In particular, the capability of human neutrophils to kill the organism was reported to be even greater than that of monocytes $(52,55)$. In vitro, mouse neutrophils appear to move toward $C$. neoformans and then rapidly internalize the yeast (56). Complement C5a-C5aR signaling was essential for phagocytosis of $C$. neoformans by neutrophils by guiding their migration to neutrophils and enhancing surface expression of CD11b (56). Furthermore, the p38 MAPK pathway, but not the Erk pathway, was critically involved in C5a-C5aR-mediated chemotaxis of neutrophils during their killing of C. neoformans (56). These in vitro observations encouraged us to address how neutrophils dynamically interact with $C$. neoformans which were arrested in the brain vasculature (23). With the use of IVM, we demonstrated that neutrophils crawled to the yeast cells that had been arrested in the brain microvasculature. Interestingly, crawling neutrophils recognized and interacted with the yeast, resulting in internalization of C. neoformans. During the interactions of neutrophils with the yeast, morphologic alterations of neutrophils, including deploying pseudopodia, were observed. Internalization of C. neoformans by neutrophils in the brain vasculature could be completed within a few minutes. Following ingestion of C. neoformans, neutrophils were seen to crawl again along the vessel wall and eventually to be released into the blood flow, resulting in a direct removal of the arrested $C$. neoformans from the brain vasculature. Depletion of neutrophils enhanced brain fungal burden (23), while enhancing the recruitment of neutrophils improved intravascular clearance of $C$. neoformans in the brain (57). Further studies demonstrated that C. neoformans infection led to enhanced expression of the adhesion molecule, Mac-1, on neutrophils, and ICAM-1 on brain endothelial cells. Complement C3 was critically involved in the recognition of $C$. neoformans by neutrophils and subsequent clearance of the organism from the brain (23). These results revealed that neutrophils are able to remove C. neoformans that had been arrested in the brain microvasculature in a "vacuumcleaner" type of behavior. Given that neutrophils are usually considered to kill microorganisms at the infection site, the finding of the direct removal of $C$. neoformans by neutrophils from its arrested site may represent a novel mechanism of host defense in the brain (23). In this respect, neutrophils have been recently shown to "sweep up" bacteria arrested on the walls of an infected body cavity or blood vessel, but not fluid-borne bacteria in a zebrafish model (58).

Recently, a live-imaging model based on zebrafish larvae has been established to study the interactions of $C$. neoformans with innate immune cells and its migration to the brain $(24,25)$. The zebrafish $C$. neoformans platform provides a visually and genetically accessible vertebrate model system for infection of C. neoformans. It was shown that zebrafish macrophages rapidly phagocytosed the majority of $C$. neoformans cells following injection of the yeast via the caudal vein (25). Depletion of macrophages significantly enhanced the fungal burden in zebrafish, demonstrating that macrophages are essential to protect zebrafish from disease progression $(24,25)$. However, macrophages preferentially ingested $C$. neoformans with smaller polysaccharide capsules, and since the capsule size greatly increased over $24 \mathrm{~h}$ of infection, this markedly limited further phagocytosis (24). In addition, proliferation of C. neoformans within macrophages and non-lytic exocytosis of the yeast from macrophages were observed in zebrafish (24). Live 
imaging demonstrated that $C$. neoformans is able to penetrate the zebrafish brain. There was a positive correlation between the burden of organisms in cranial vessels versus invasion into the brain parenchyma (25). The C. neoformans fnx $1 \Delta$ mutant, which is deficient in a multidrug resistance-like protein, was shown to have a deficiency in transmigration across the mouse BBB and reduced microvascular entrapment and transcytosis across immortalized human brain capillary endothelial cells in vitro (59). Interestingly, the fnx $1 \Delta$ mutant also demonstrated defective invasion of brain parenchyma of zebrafish (25). Using IVM, these studies are just beginning to enhance our understanding of the spacial and temporal aspects and the role of different cell types in pathogenesis and host defense to C. neoformans.

\section{BRAIN INFECTION WITH C. ALBICANS}

Candida albicans is a commensal organism and a common constituent of the normal mucosal flora. As the most common fungal pathogen of humans, overgrowth causes thrush. However, translocation of the yeast cells from the mucosal surface into the systemic circulation causes potentially life-threatening disease, particularly in post-surgical and critically ill patients, which is associated with approximately $35 \%$ death rate $(60,61)$. During this disease, the bloodborne organisms can spread to virtually all organs of the body. Although the kidney is the primary target of this organism during disseminated candidiasis, brain infection is found in approximately one-half of patients with systemic candidiasis at autopsy (62-64). In addition, C. albicans has also been reported to cause meningoencephalitis without systemic infection in healthy individuals (65).

To invade the brain parenchyma, circulating C. albicans cells must adhere and cross the BBB. Early work had shown that C. albicans is able to penetrate a monolayer of human brain endothelial cells cultured in vitro via a transcellular pathway (66). It was later demonstrated that C. albicans invasion of brain endothelial cells is mediated by the fungal invasins Als3 and Ssa1 (67). Als3 binds to the gp96 heat shock protein, a unique receptor that is expressed specifically on brain endothelium, promoting endothelial transcytosis by the fungus (67).

Recently, Navarathna et al. studied brain infection by C. albicans in a mouse model using IVM (26). They observed sporadic entry of C. albicans into the brain parenchyma as early as 30 min after intravenous inoculation. In this model, the authors did not observeleak of gadolinium diethylenetriaminepentaacetic acid (Gd-DTPA) into the brain 30 min after intravenous administration as examined by MRI, suggesting that brain invasion by $C$. albicans initially occurs without gross disruption of the $\mathrm{BBB}$. However, IVM performed 3 days post-infection revealed significant accumulation of both yeast and filamentous forms of C. albicans in the meninges and parenchyma. At that time, leak of Gd-DTPA was observed, indicating damage of the BBB. The brain became heavily inflamed at sites of $C$. albicans invasion. Thus, it is conceivable that permeability of the BBB was caused by leukocyte infiltration. In addition, Candida filament elongation was observed in the brain. Interestingly, most of the yeast cells outside of the vasculature showed highly dynamic movement that could be explained by the movement of phagocytosed organisms within motile phagocytic cells. By contrast, hyphal cells showed only slow invasion based on hyphal extension.

\section{BRAIN INFECTION WITH P. BRASILIENSIS}

Paracoccidioides brasiliensis is an etiologic agent of paracoccidioidomycosis, an important systemic mycosis in Latin America, with the greatest number of patients in Brazil, Venezuela, and Argentina (68). The infection is usually acquired by the respiratory system probably by inhalation of airborne conidia of $P$. brasiliensis $(69,70)$. Following infection, the conidia transform into yeast in the lungs. $P$. brasiliensis can cause disease in immunocompetent hosts, although immunosuppression increases the severity of infection. The yeast cells can be disseminated from the infected lung into other organs such as adrenal glands and brain $(70,71)$. In the last decade, brain infection has been reported more commonly, affecting approximately $12.5 \%$ of cases (70). However, it is unknown how the fungus arrests and migrates into the brain parenchyma across the BBB.

Recently, Pedroso et al. used IVM to examine trafficking of leukocytes in the brain in a murine model of neuroparacoccidioidomycosis (20). Following infection with P. brasiliensis by the intracranial route, mice showed clinical signs of progressive infection starting on day 7 post-inoculation. IVM of the brain pial microvasculature revealed a significant increase in leukocyte rolling 2 and 4 weeks post-infection and in adhesion 1, 2, and 4 weeks post-infection. The enhanced recruitment of leukocytes was associated with a significant increase in the brain concentration of chemokines, particularly CXCL9, suggesting a role for these molecules in the inflammatory and immune response against the fungi. The lesions were not restricted to the site of inoculation and disseminated to other sites of the brain including the cerebellum. Neutrophils and macrophages were increased in the brain as determined by the myeloperoxidase and $N$-acetyl-bD-glucosaminidase activity in the brain tissues.

\section{CONCLUDING REMARKS}

Fungal meningoencephalitis is a grave illness associated with high mortality, even with the best available antifungal treatment. Understanding the mechanisms involved in arrest and invasion of the brain by fungi and the interactions with immune cells is fundamental to our knowledge of the pathogenesis of the disease. With the use of IVM, brain infections by fungi, including C. neoformans $(5,23)$, C. albicans (26), and P. brasiliensis (20), have been recently investigated in real time. In particular, we have shown that $C$. neoformans is mechanically trapped in the brain vasculature (5). IVM may provide a powerful tool to determine whether in vitro findings implicating interactions between CD44 and hyaluronic acid $(7,8)$, or adherence of phagocytosed cells (Trojan Horse) also occur $(11,12)$ through the use of transgenic mice. Although neutrophils are able to recognize and remove the arrested C. neoformans from the brain vasculature (23), organisms were seen to cross the vessel wall with contribution of cryptococcal urease (5). IVM may provide a powerful tool to investigate the role of metalloprotease (9), and phospholipase (10) in brain invasion via transcytosis through the use of deletion 
mutants. The role of host immune cells in brain injury has been implicated in studies using C. albicans, and chemokines have been implicated by studies of $P$. brasiliensis $(20,26)$. In summary, recent advances in microscopy and the availability of antibody labeling in vivo, transgenic reporter mice and mice with targeted gene disruptions provide a powerful tool to examine fungal brain infections under more relevant physiologic conditions. It is expected that exciting findings regarding spacial and temporal aspects of pathogenic and immune mechanisms in fungal brain infections will be obtained with the application of IVM in the future, and that these insights may lead to new therapies.

\section{REFERENCES}

1. Kim KS. Mechanisms of microbial traversal of the blood-brain barrier. Nat Rev Microbiol (2008) 6(8):625-34. doi:10.1038/nrmicro1952

2. Owens T, Bechmann I, Engelhardt B. Perivascular spaces and the two steps to neuroinflammation. J Neuropathol Exp Neurol (2008) 67(12):1113-21. doi:10.1097/NEN.0b013e31818f9ca8

3. Engelhardt B, Sorokin L. The blood-brain and the blood-cerebrospinal fluid barriers: function and dysfunction. Semin Immunopathol (2009) 31(4):497511. doi:10.1007/s00281-009-0177-0

4. Shi M, Colarusso P, Mody CH. Real-time in vivo imaging of fungal migration to the central nervous system. Cell Microbiol (2012) 14(12):1819-27. doi: $10.1111 / \mathrm{cmi} .12027$

5. Shi M, Li SS, Zheng C, Jones GJ, Kim KS, Zhou H, et al. Real-time imaging of trapping and urease-dependent transmigration of Cryptococcus neoformans in mouse brain. J Clin Invest (2010) 120(5):1683-93. doi:10.1172/JCI41963

6. Chang YC, Stins MF, McCaffery MJ, Miller GF, Pare DR, Dam T, et al. Cryptococcal yeast cells invade the central nervous system via transcellular penetration of the blood-brain barrier. Infect Immun (2004) 72(9):4985-95. doi:10.1128/IAI.72.9.4985-4995.2004

7. Jong A, Wu CH, Gonzales-Gomez I, Kwon-Chung KJ, Chang YC, Tseng HK, et al. Hyaluronic acid receptor CD44 deficiency is associated with decreased Cryptococcus neoformans brain infection. JBiol Chem (2012) 287(19): 15298-306. doi:10.1074/jbc.M112.353375

8. Jong A, Wu CH, Shackleford GM, Kwon-Chung KJ, Chang YC, Chen HM, et al. Involvement of human CD44 during Cryptococcus neoformans infection of brain microvascular endothelial cells. Cell Microbiol (2008) 10(6):1313-26. doi:10.1111/j.1462-5822.2008.01128.x

9. Vu K, Tham R, Uhrig JP, Thompson GR III, Na Pombejra S, Jamklang M, et al. Invasion of the central nervous system by Cryptococcus neoformans requires a secreted fungal metalloprotease. MBio (2014) 5(3):e1101-14. doi:10.1128/ mBio.01101-14

10. Maruvada R, Zhu L, Pearce D, Zheng Y, Perfect J, Kwon-Chung KJ, et al. Cryptococcus neoformans phospholipase B1 activates host cell Racl for traversal across the blood-brain barrier. Cell Microbiol (2012) 14(10):1544-53. doi:10.1111/j.1462-5822.2012.01819.x

11. Charlier C, Nielsen K, Daou S, Brigitte M, Chretien F, Dromer F. Evidence of a role for monocytes in dissemination and brain invasion by Cryptococcus neoformans. Infect Immun (2009) 77(1):120-7. doi:10.1128/IAI.01065-08

12. Sorrell TC, Juillard PG, Djordjevic JT, Kaufman-Francis K, Dietmann A, Milonig A, et al. Cryptococcal transmigration across a model brain blood-barrier: evidence of the Trojan horse mechanism and differences between Cryptococcus neoformans var. grubii strain H99 and Cryptococcus gattii strain R265. Microbes Infect (2016) 18(1):57-67. doi:10.1016/j.micinf.2015.08.017

13. Bryan AM, Del Poeta M, Luberto C. Sphingolipids as regulators of the phagocytic response to fungal infections. Mediators Inflamm (2015) 2015:640540. doi:10.1155/2015/640540

14. Bariola JR, Perry P, Pappas PG, Proia L, Shealey W, Wright PW, et al Blastomycosis of the central nervous system: a multicenter review of diagnosis and treatment in the modern era. Clin Infect Dis (2010) 50(6):797-804. doi:10.1086/650579

15. Chakrabarti A. Epidemiology of central nervous system mycoses. Neurol India (2007) 55(3):191-7. doi:10.4103/0028-3886.35679

\section{AUTHOR CONTRIBUTIONS}

All authors listed have made substantial, direct, and intellectual contribution to the work and approved it for publication.

\section{FUNDING}

This work was supported by U.S. National Institutes of Health National Institute of Allergy and Infectious Diseases Grant AI115086A (to MS) and Canadian Institute for Health Research Grant 247301 (to CM).

16. Gottfredsson M, Perfect JR. Fungal meningitis. Semin Neurol (2000) 20(3):307-22. doi:10.1055/s-2000-9394

17. Kleinschmidt-DeMasters BK. Central nervous system aspergillosis: a 20-year retrospective series. Hum Pathol (2002) 33(1):116-24. doi:10.1053/hupa. 2002.30186

18. Liu TB, Perlin DS, Xue C. Molecular mechanisms of cryptococcal meningitis. Virulence (2012) 3(2):173-81. doi:10.4161/viru.18685

19. Murthy JM. Fungal infections of the central nervous system: the clinical syndromes. Neurol India (2007) 55(3):221-5. doi:10.4103/0028-3886.35682

20. Pedroso VS, Vilela MC, Santos PC, Cisalpino PS, Rachid MA, Teixeira AL. Traffic of leukocytes and cytokine up-regulation in the central nervous system in a murine model of neuroparacoccidioidomycosis. Mycopathologia (2013) 176(3-4):191-9. doi:10.1007/s11046-013-9679-3

21. Scully EP, Baden LR, Katz JT. Fungal brain infections. Curr Opin Neurol (2008) 21(3):347-52. doi:10.1097/WCO.0b013e3282fee95b

22. Park BJ, Wannemuehler KA, Marston BJ, Govender N, Pappas PG, Chiller TM. Estimation of the current global burden of cryptococcal meningitis among persons living with HIV/AIDS. AIDS (2009) 23(4):525-30. doi:10.1097/ QAD.0b013e328322ffac

23. Zhang M, Sun D, Liu G, Wu H, Zhou H, Shi M. Real-time in vivo imaging reveals the ability of neutrophils to remove Cryptococcus neoformans directly from the brain vasculature. J Leukoc Biol (2016) 99(3):467-73. doi:10.1189/ jlb.4AB0715-281R

24. Bojarczuk A, Miller KA, Hotham R, Lewis A, Ogryzko NV, Kamuyango AA, et al. Cryptococcus neoformans intracellular proliferation and capsule size determines early macrophage control of infection. Sci Rep (2016) 6:21489. doi:10.1038/srep21489

25. Tenor JL, Oehlers SH, Yang JL, Tobin DM, Perfect JR. Live imaging of host-parasite interactions in a zebrafish infection model reveals cryptococcal determinants of virulence and central nervous system invasion. MBio (2015) 6(5):e1425-1415. doi:10.1128/mBio.01425-15

26. Navarathna DH, Munasinghe J, Lizak MJ, Nayak D, McGavern DB, Roberts DD. MRI confirms loss of blood-brain barrier integrity in a mouse model of disseminated candidiasis. NMR Biomed (2013) 26(9):1125-34. doi:10.1002/ nbm. 2926

27. Dutrochet H. Recherches Anatomiques et Physiologiques sur la Structure Intime des Animaux et des Vegetaux, et sur Leur Motilite. Paris: Bailliere et fils (1824)

28. Helmchen F, Denk W. Deep tissue two-photon microscopy. Nat Methods (2005) 2(12):932-40. doi:10.1038/nmeth818

29. Graf R, Rietdorf J, Zimmermann T. Live cell spinning disk microscopy. Adv Biochem Eng Biotechnol (2005) 95:57-75. doi:10.1007/b102210

30. Murray JM. Methods for imaging thick specimens: confocal microscopy, deconvolution, and structured illumination. Cold Spring Harb Protoc (2011) 2011(12):1399-437. doi:10.1101/pdb.top066936

31. Conchello JA, Lichtman JW. Optical sectioning microscopy. Nat Methods (2005) 2(12):920-31. doi:10.1038/nmeth815

32. Ustione A, Piston DW. A simple introduction to multiphoton microscopy. J Microsc (2011) 243(3):221-6. doi:10.1111/j.1365-2818.2011.03532.x

33. Yang G, Pan F, Parkhurst CN, Grutzendler J, Gan WB. Thinned-skull cranial window technique for long-term imaging of the cortex in live mice. Nat Protoc (2010) 5(2):201-8. doi:10.1038/nprot.2009.222

34. Lionakis MS, Swamydas M, Fischer BG, Plantinga TS, Johnson MD, Jaeger M, et al. CX3CR1-dependent renal macrophage survival promotes Candida 
control and host survival. J Clin Invest (2013) 123(12):5035-51. doi:10.1172/ JCI71307

35. Voelz K, Johnston SA, Rutherford JC, May RC. Automated analysis of cryptococcal macrophage parasitism using GFP-tagged cryptococci. PLoS One (2010) 5(12):e15968. doi:10.1371/journal.pone.0015968

36. Albelda SM, Muller WA, Buck CA, Newman PJ. Molecular and cellular properties of PECAM-1 (endoCAM/CD31): a novel vascular cell-cell adhesion molecule. J Cell Biol (1991) 114(5):1059-68. doi:10.1083/jcb.114.5.1059

37. Moriarty TJ, Norman MU, Colarusso P, Bankhead T, Kubes P, Chaconas G. Real-time high resolution 3D imaging of the Lyme disease spirochete adhering to and escaping from the vasculature of a living host. PLoS Pathog (2008) 4(6):e1000090. doi:10.1371/journal.ppat.1000090

38. Phillipson M, Heit B, Colarusso P, Liu L, Ballantyne CM, Kubes P. Intraluminal crawling of neutrophils to emigration sites: a molecularly distinct process from adhesion in the recruitment cascade. J Exp Med (2006) 203(12):2569-75. doi:10.1084/jem.20060925

39. McDonald B, Pittman K, Menezes GB, Hirota SA, Slaba I, Waterhouse CC, et al. Intravascular danger signals guide neutrophils to sites of sterile inflammation. Science (2010) 330(6002):362-6. doi:10.1126/science.1195491

40. Motoike T, Loughna S, Perens E, Roman BL, Liao W, Chau TC, et al. Universal GFP reporter for the study of vascular development. Genesis (2000) 28(2):7581. doi:10.1002/1526-968X(200010)28:2<75::AID-GENE50>3.0.CO;2-S

41. Zhou H, Andonegui G, Wong CH, Kubes P. Role of endothelial TLR4 for neutrophil recruitment into central nervous system microvessels in systemic inflammation. J Immunol (2009) 183(8):5244-50. doi:10.4049/ jimmunol.0901309

42. Zhou H, Lapointe BM, Clark SR, Zbytnuik L, Kubes P. A requirement for microglial TLR4 in leukocyte recruitment into brain in response to lipopolysaccharide. JImmunol (2006) 177(11):8103-10. doi:10.4049/ jimmunol.177.11.8103

43. Faust N, Varas F, Kelly LM, Heck S, Graf T. Insertion of enhanced green fluorescent protein into the lysozyme gene creates mice with green fluorescent granulocytes and macrophages. Blood (2000) 96(2):719-26.

44. Auffray C, Fogg D, Garfa M, Elain G, Join-Lambert O, Kayal S, et al. Monitoring of blood vessels and tissues by a population of monocytes with patrolling behavior. Science (2007) 317(5838):666-70. doi:10.1126/science.1142883

45. Geissmann F, Jung S, Littman DR. Blood monocytes consist of two principal subsets with distinct migratory properties. Immunity (2003) 19(1):71-82. doi:10.1016/S1074-7613(03)00174-2

46. Jung S, Aliberti J, Graemmel P, Sunshine MJ, Kreutzberg GW, Sher A, et al. Analysis of fractalkine receptor CX(3)CR1 function by targeted deletion and green fluorescent protein reporter gene insertion. Mol Cell Biol (2000) 20(11):4106-14. doi:10.1128/MCB.20.11.4106-4114.2000

47. Kwon-Chung KJ, Sorrell TC, Dromer F, Fung E, Levitz SM. Cryptococcosis: clinical and biological aspects. Med Mycol (2000) 38(Suppl 1):205-13. doi:10.1080/mmy.38.s1.205.213

48. Sabiiti W, May RC. Capsule independent uptake of the fungal pathogen Cryptococcus neoformans into brain microvascular endothelial cells. PLoS One (2012) 7(4):e35455. doi:10.1371/journal.pone.0035455

49. Vu K, Weksler B, Romero I, Couraud PO, Gelli A. Immortalized human brain endothelial cell line HCMEC/D3 as a model of the blood-brain barrier facilitates in vitro studies of central nervous system infection by Cryptococcus neoformans. Eukaryot Cell (2009) 8(11):1803-7. doi:10.1128/EC.00240-09

50. Liu TB, Kim JC, Wang Y, Toffaletti DL, Eugenin E, Perfect JR, et al. Brain inositol is a novel stimulator for promoting Cryptococcus penetration of the blood-brain barrier. PLoS Pathog (2013) 9(4):e1003247. doi:10.1371/journal. ppat. 1003247

51. Kolaczkowska E, Kubes P. Neutrophil recruitment and function in health and inflammation. Nat Rev Immunol (2013) 13(3):159-75. doi:10.1038/nri3399

52. Diamond RD, Root RK, Bennett JE. Factors influencing killing of Cryptococcus neoformans by human leukocytes in vitro. J Infect Dis (1972) 125(4):367-76. doi:10.1093/infdis/125.4.367

53. Kozel TR, Highison B, Stratton CJ. Localization on encapsulated Cryptococcus neoformans of serum components opsonic for phagocytosis by macrophages and neutrophils. Infect Immun (1984) 43(2):574-9.

54. Qureshi A, Subathra M, Grey A, Schey K, Del Poeta M, Luberto C. Role of sphingomyelin synthase in controlling the antimicrobial activity of neutrophils against Cryptococcus neoformans. PLoS One (2010) 5(12):e15587. doi:10.1371/ journal.pone.0015587
55. Miller MF, Mitchell TG. Killing of Cryptococcus neoformans strains by human neutrophils and monocytes. Infect Immun (1991) 59(1):24-8.

56. Sun D, Zhang M, Liu G, Wu H, Zhu X, Zhou H, et al. Real-time imaging of interactions of neutrophils with Cryptococcus neoformans demonstrates a crucial role of complement C5a-C5aR signaling. Infect Immun (2015) 84(1):216-29. doi:10.1128/IAI.01197-15

57. Sun D, Zhang M, Liu L, Wu H, Li C, Zhou H, et al. Intravascular clearance of disseminating Cryptococcus neoformans in the brain can be improved by enhancing neutrophil recruitment in mice. Eur J Immunol (2016) 46(7):170414. doi:10.1002/eji.201546239

58. Colucci-Guyon E, Tinevez JY, Renshaw SA, Herbomel P. Strategies of professional phagocytes in vivo: unlike macrophages, neutrophils engulf only surface-associated microbes. J Cell Sci (2011) 124(Pt 18):3053-9. doi:10.1242/ jcs. 082792

59. Tseng HK, Liu CP, Price MS, Jong AY, Chang JC, Toffaletti DL, et al. Identification of genes from the fungal pathogen Cryptococcus neoformans related to transmigration into the central nervous system. PLoS One (2012) 7(9):e45083. doi:10.1371/journal.pone.0045083

60. Horn DL, Neofytos D, Anaissie EJ, Fishman JA, Steinbach WJ, Olyaei AJ, et al. Epidemiology and outcomes of candidemia in 2019 patients: data from the prospective antifungal therapy alliance registry. Clin Infect Dis (2009) 48(12):1695-703. doi:10.1086/599039

61. Wisplinghoff H, Bischoff T, Tallent SM, Seifert H, Wenzel RP, Edmond MB. Nosocomial bloodstream infections in US hospitals: analysis of 24,179 cases from a prospective nationwide surveillance study. Clin Infect Dis (2004) 39(3):309-17. doi:10.1086/421946

62. Parker JC Jr, McCloskey JJ, Lee RS. Human cerebral candidosis - a postmortem evaluation of 19 patients. Hum Pathol (1981) 12(1):23-8. doi:10.1016/ S0046-8177(81)80238-9

63. Pendlebury WW, Perl DP, Munoz DG. Multiple microabscesses in the central nervous system: a clinicopathologic study. J Neuropathol Exp Neurol (1989) 48(3):290-300. doi:10.1097/00005072-198905000-00006

64. Sanchez-Portocarrero J, Perez-Cecilia E, Corral O, Romero-Vivas J, Picazo JJ. The central nervous system and infection by Candida species. Diagn Microbiol Infect Dis (2000) 37(3):169-79. doi:10.1016/S0732-8893(00)00140-1

65. Borha A, Parienti JJ, Emery E, Coskun O, Khouri S, Derlon JM. [Candida albicans cerebral granuloma in an immunocompetent patient. A case report]. Neurochirurgie (2009) 55(1):57-62. doi:10.1016/j.neuchi.2008.06.001

66. Jong AY, Stins MF, Huang SH, Chen SH, Kim KS. Traversal of Candida albicans across human blood-brain barrier in vitro. Infect Immun (2001) 69(7):4536-44. doi:10.1128/IAI.69.7.4536-4544.2001

67. Liu Y, Mittal R, Solis NV, Prasadarao NV, Filler SG. Mechanisms of Candida albicans trafficking to the brain. PLoS Pathog (2011) 7(10):e1002305. doi:10.1371/journal.ppat.1002305

68. de Oliveira HC, Assato PA, Marcos CM, Scorzoni L, de Paula ESAC, Da Silva Jde F, et al. Paracoccidioides-host interaction: an overview on recent advances in the paracoccidioidomycosis. Front Microbiol (2015) 6:1319. doi:10.3389/ fmicb.2015.01319

69. McEwen JG, Bedoya V, Patino MM, Salazar ME, Restrepo A. Experimental murine paracoccidiodomycosis induced by the inhalation of conidia. J Med Vet Mycol (1987) 25(3):165-75. doi:10.1080/02681218780000231

70. Pedroso VS, Vilela Mde C, Pedroso ER, Teixeira AL. [Paracoccidioidomycosis compromising the central nervous system: a systematic review of the literature]. Rev Soc Bras Med Trop (2009) 42(6):691-7. doi:10.1590/S003786822009000600016

71. Paniago AM, de Oliveira PA, Aguiar ES, Aguiar JI, da Cunha RV, Leme LM, et al. Neuroparacoccidioidomycosis: analysis of 13 cases observed in an endemic area in Brazil. Trans R Soc Trop Med Hyg (2007) 101(4):414-20. doi:10.1016/j.trstmh.2006.07.006

Conflict of Interest Statement: The authors declare that the research was conducted in the absence of any commercial or financial relationships that could be construed as a potential conflict of interest.

Copyright $\odot 2016$ Shi and Mody. This is an open-access article distributed under the terms of the Creative Commons Attribution License (CC BY). The use, distribution or reproduction in other forums is permitted, provided the original author(s) or licensor are credited and that the original publication in this journal is cited, in accordance with accepted academic practice. No use, distribution or reproduction is permitted which does not comply with these terms. 